\title{
ENSEÑANZA DE PROCESOS DE PENSAMIENTO: METODOLOGÍA, METACOGNICIÓN Y TRANSFERENCIAS
}

\section{Teaching of thinking processes: methodology, metacognition and transference}

por

$\underline{\text { Article record }}$

$\underline{\text { HTML format }}$

\author{
Juan Francisco Martín Izard
}

Ficha del artículo

Formato HTML

\begin{abstract}
We analyze the importance of teaching with the correct methodology in thinking processes. We also present an empirical study which shows the importance of teachers' familiarity with these methodologies of thinking processes.
\end{abstract}

\section{Keywords}

Thinking processes, metacognition, transference.

\section{Resumen}

Analizamos la importancia de una correcta metodología en la enseñanza de los procesos de pensamiento. Presentamos también un estudio empírico en el que se intentará poner de manifiesto la importancia de que el profesor conozca las metodologías mencionadas

\section{Descriptores}

Procesos de pensamiento, metacognición, transferencia

\section{Introducción.}

En este artículo se pretenden algunos datos e informaciones que pongan de manifiesto la importancia de una correcta metodología en la enseñanza de los procesos de pensamiento. Para ello vamos a revisar cómo se ha llegado a otorgar especial importancia a estos aspectos , tanto desde el ámbito de la investigación como desde la práctica educativa en los centros escolares, pasando por las indicaciones legislativas al respecto.
Consideramos que uno de los principales problemas con los que el profesional de la educación se encuentra ante la enseñanza de procesos de pensamiento es la carencia de una correcta metodología que le facilite trabajar estrategias metacognitivas y transferirlas a otros ámbitos de conocimiento. En este sentido haremos un esbozo de las metodologías que consideramos más adecuadas para este fin.

Finalmente mostraremos, a modo de ejemplo, un estudio empírico en el que se intenta-

\footnotetext{
a Dpto.de Didáctica, Organización y Métodos de Investigación (Universidad de Salamanca)
} 
rá poner de manifiesto la importancia de que el profesor conozca las metodologías mencionadas.

\section{1.- La necesidad de enseñar procesos de pensamiento: un breve recorrido histórico y algunos problemas detec- tados.}

Desde la Reforma educativa suscitada a partir de la promulgación de la LOGSE (1992), la enseñanza de los procesos cobra especial relevancia frente a tradicionales formas de enseñanza centradas exclusivamente en lo que a partir de esos momentos se llamó conocimiento declarativo. El conocimiento procesual y procedimental se considera de importancia para el desarrollo integral del alumno. Se empieza a debatir a principios de los años ochenta, antes de la reforma de la LOGSE, acerca de la relativa importancia de la enseñanza de conceptos frente a la enseñanza de procedimientos. Hasta este momento la enseñanza se hacía cargo fundamentalmente del conocimiento declarativo, de los conceptos. El interés por la enseñanza de los procedimientos surge de una preocupación creciente en los setenta y ochenta del profesorado, fundamentalmente de secundaria, que manifestaban las dificultades de los alumnos para estudiar las materias y enfrentarse a nuevos conocimientos, sobre todo cuando estos requieren estrategias de resolución de problemas.

La respuesta del Ministerio de Educación y Ciencia fue clara ante la reforma educativa. Uno se los pilares sobre los que se asentaba la personalización de la enseñanza era conseguir la autonomía de aprendizaje de los alumnos (promover recursos que les permitan enfrentarse a los aprendizajes de manera independiente). De esta forma, en el Libro Blanco para la Reforma del Sistema Educativo se afirma:

"Las personas se verán cada vez más enfrentadas a cambios que afectan a sus capacidades como trabajadores y su capacidad de respuesta dependerá esencialmente de sus niveles educativos. (...) En estas condiciones previsibles resulta indispensable una formación de base más alta que la proporcionada por la actual E.G.B., y también una educación más polivalente y flexible, que permita a las personas encarar nuevas situaciones con garantía de éxito” (M.E.C., 1989a, p.88).

Insistiendo en esta idea en el posterior documento sobre el Diseño Curricular Base se afirma que será necesario:

"Es absolutamente preciso hacer consciente al alumno de los procesos que se emplean en la elaboración de conocimientos, facilitándole por todos los medios la reflexión metacognitiva sobre las habilidades de conocimiento, los procesos cognitivos, el control y la planificación de la propia actuación y de los otros, la toma de decisiones y la comprobación de resultados” (M.E.C., 1989b, p.99).

El interrogante, que se menifestaba en términos de ¿enseñamos contenidos o enseñamos a aprender?, quedaba aparentemente resuelto concediéndole la importancia que hasta el momento no tenía el "aprender a aprender” y el "aprender a pensar”. Esto se tradujo en una gran expansión de materiales que afrontaban este nuevo reto en dos frentes (Monereo y otros 1996) por una parte las técnicas de estudio, y por otra los programas de aprender a pensar. Es en estos momentos cuando nos preocupa especialmente cómo hacer llegar al alumno este "nuevo" tipo de conocimiento que no sólo enseña conceptos y hechos, sino también procesos y procedimientos para elaborar estos conceptos y para resolver problemas. A partir de estos momentos empiezan a desarrollarse diversas estrategias y herramientas de enseñanza centradas en el aprendizaje de los procesos de pensamiento. Aparecen así en los ambientes escolares variados programas de los que genéricamente se les llamó de dos formas: bien programas de “enseñar a pensar" en clara alusión a la enseñanza de procesos , o bien programas para la "mejora de la inteligencia" 
haciendo referencia al aspecto psicológico que pretendían mejorar.

En un primer momento, anterior incluso a la mencionada reforma, estos programas aparecen como "material especial" de importación, de difícil acceso y más complicado manejo. Efectivamente, para el uso de estos programas es necesaria una formación previa, si bien no tan profunda e "inaccesible" como en aquellos momentos se pensaba.

No exentos de cierto "halo de misterio" comienzan alrededor de los años 80 a difundirse estos programas y comienzan a plantearse en nuestro país investigaciones y estudios al respecto (Calero, 1986; Prieto, 1989,1992; Molina y Arraiz, 1993; Martínez Beltran y otros, 1990; Beltrán 1993; Martín Izard, 1995; Yuste, 1997). En estos momentos las expectativas que los educadores pusieron sobre estos programas fueron, cuanto menos, generosas.

Una vez superada esta primera fase, cuando ya se habían realizado suficientes estudios en nuestro país, eran suficientemente conocidos estos programas. Con la ayuda de la reforma educativa planteada a principios de los 90, comienzan a editarse en nuestro entorno. Primero aquellos que eran sobradamente conocidos y ya probados en otros contextos como el Programa de Enriquecimiento Instrumental de Feuerstein (1992) o el Proyecto inteligencia "Harvard” (Megía 1993), por citar dos de los más utilizados. Posteriormente comienzan a publicarse programas creados en nuestro país, algunos con mejor soporte teórico y experimental que otros, hasta el punto de "saturar" el mercado.

Esta expansión, junto con las elevadas expectativas de los educadores, propició que en muchas ocasiones los programas se aplicaran precipitadamente, sin una validación y experimentación suficiente y sin una necesaria formación previa por parte de los aplicadores. En estas circunstancias los resultados no fueron los esperados y el desencanto de nu- merosos profesores se hizo patente al no encontrar lo que buscaban.

En este punto de la cuestión, los investigadores ya habíamos advertido que estos programas no sirven para arreglar todas las situaciones problemáticas en el ámbito escolar, sirven para lo que sirven; y además, deben de emplearse correctamente si queremos que se vean cumplidos sus objetivos.

Varios eran los problemas que se detectaron en la aplicación de los programas. En este artículo nos interesan especialmente dos:

- Los programas requieren de una metodología concreta, de un "modus operandi" que puede que no difiera mucho de la actuación normal del profesor, pero que sí requiere de una formación previa y, frecuentemente, de un cambio de actitud del profesor.

- La transferencia de los aprendizajes realizados en los programas no es automática. Muchos de estos programas se denominan "libres de contenido", aludiendo a la ausencia de contenidos curriculares habituales en las aulas. Si bien este aspecto facilita el trabajo en un primer momento sobre los procesos de pensamiento, hace que se tenga que poner mucha atención y dedicar un gran esfuerzo en que estos aprendizajes se utilicen tanto en las áreas del curriculum como en la vida cotidiana de los alumnos.

Pensamos que ambos problemas, metodología y transferencia, se encuentran estrechamente ligados, de tal forma que una metodología adecuada al enseñar procesos de pensamiento facilita al aprendiz la realización de las necesarias trasferencias para que estos aprendizajes sean eficaces. Pero para llegar a estas conclusiones quizá sea necesario previamente aclarar algunas concepciones, desde nuestro punto de vista erróneas, que se han ido generando en este periodo que acabamos de comentar.

\section{2.- Algunas dicotomías falsas.}


Son varias las dicotomías que suelen aparecer en los debates que pretenden clarificar la importancia de enseñar a pensar. Como acabamos de ver en el apartado anterior, la primera dicotomía que apareció es si se deben enseñar contenidos declarativos o procedimentales. Esta dicotomía suscitó, como hemos comentado, enriquecedores debates en los años 80 ya superados, según nuestro parecer y cuya solución sólo sugiere un comentario: como en tantas otras cosas, según dice el dicho popular, "en el punto medio está la verdad”.

La dicotomía que se presenta ahora, una vez que se han desarrollado y aplicado innumerables programas, viene formulada por la cuestión de si se enseñan dentro del curriculum o fuera de él, es decir, dentro de los programas de las asignaturas y referidos a los contenidos propios de las materias, o como un curriculum independiente, desarrollando habilidades y destrezas que luego el alumno habrá de transferir. Desde nuestro punto de vista esta cuestión no está todavía suficientemente debatida.

En un primer momento del desarrollo de estos programas se trabajaron como aspectos separados de las materias. Tanto técnicas de estudio como aprender a pensar se ejercitaba con materiales diferentes a los habitualmente empleados por los profesores en sus asignaturas. Proliferan en estos momentos los programas específicos, concretamente en "enseñar a pensar" los programas denominados "libres de contenido". De esta forma los alumnos tenían en sus horarios espacios reservados para este otro tipo de actividad.

Los resultados no fueron los esperados ya que, como las investigaciones pusieron de manifiesto, el conocimiento de una estrategia o una técnica es necesario, pero no suficiente para que ésta se utilice adecuadamente (Wittrock, 1985; Pressley, 1990, Monereo y otros, 1994; Prieto, 1992; Mayor y otros, 1993).
De esta forma, en los últimos años la tendencia clara es que no se puede enseñar a aprender y a pensar independientemente de los contenidos sobre los que se aprende (Monereo y otros, 1996). Desde nuestro punto de vista, si bien esta afirmación es cierta en sí misma, es necesario matizarla, dado que corremos el riesgo de que ocurra, como es frecuente en educación, que actuemos según el movimiento del péndulo y nos situemos en un extremo u otro sin contemplar las posibilidades que ofrecen los puntos intermedios.

Evidentemente ambas posturas opuestas pueden presentar sus ventajas e inconvenientes. Vamos a analizar un poco más profundamente estos extremos de la dicotomía para ver si podemos situarnos en un punto intermedio.

Pensemos primero en la enseñanza de estas técnicas, estrategias o procesos al margen de los contenidos de las materias. El inconveniente mayor ya lo hemos apuntado: no se produce una trasferencia automática con lo que el alumno aprende a pensar sobre los contenidos de los programas. O quizá deberíamos decir los "no contenidos" ya que se presume que los programas son "libres de contenido". Esta presunta "libertad de contenidos" no es totalmente cierta. Es imposible pensar "en vacío". Por muy abstracto y simbólico que sea sobre lo que estemos pensando siempre hay un contenido, bien sea pictórico, figurativo, geométrico... Estos programas de lo que sí pretenden estar libres es de contenidos académicos, se pretende cierta descarga de los contenidos culturales mayoritariamente transmitidos por el sistema escolar y reflejados en lo que conocemos como curriculum.

Pero al igual que tiene inconvenientes, esta situación también presenta sus ventajas, sobre todo en determinadas ocasiones, como es cuando trabajamos con sujetos con deficiencias debidas a su origen socio-cultural y que manifiestan una clara aversión por las tareas 
escolares, adquirida tras múltiples y repetidos fracasos. Con este tipo de sujetos es mucho más fácil el acercamiento al trabajo intelectual con unas tareas diferentes a lo que habitualmente se trabaja en clase. Hemos podido comprobar como la "motivación intrínseca" de estas tareas (Feuerstein, 1988) se pone claramente de manifiesto cuando se enfrentan a ellas, frente a una clara desmotivación por cualquier cosa que se parezca a las tareas escolares (Martín Izard,1995, 1999, 2001).

Pensemos ahora en la enseñanza de los procesos de pensamiento ligada íntimamente a la enseñanza de las materias concretas. En esta situación en cada materia del curriculum se enseña cómo se procesan las informaciones sobre estos contenidos concretos. Así por ejemplo en matemáticas se enseñarán los procesos necesarios para solucionar los problemas de geometría, aritmética, etc., y en lenguaje las cuestiones relativas a compresión y expresión escrita, morfología, sintaxis, etc. Esta situación, evidentemente, facilita que el alumno aprenda a procesar la información en cada materia, con lo que garantizamos en alguna medida el rendimiento en estos contenidos. Ahora bien, ¿̇sabrá el alumno pensar en otros contenidos que no sean estrictamente académicos?

Como vemos el problema en ambas posturas es el mismo: las transferencias y la generalización de los aprendizajes. En cualquier caso, si no trabajamos las transferencias cualquiera de ambas posturas es mala. En la primera, si enseñamos sólo con programas específicos los alumnos sólo sabrán pensar sobre los contenidos de los programas; si enseñamos a pensar ligado a contenidos académicos probablemente obtengan buenas calificaciones pero, ¿sabrán resolver un problema de su vida cotidiana?

El problema aparece en cualquier caso por desvincular lo que se aprende en una situación y en un momento concreto con el resto del mundo del aprendiz. Da igual que se aprenda sobre un programa de "aprender a pensar" o sobre matemáticas. Si estos aprendizajes sólo son eficaces en esta situación académica de poco sirven nuestros esfuerzos como educadores, ya que, cualquier caso el alumno no sabrá utilizar estos aprendizajes en su vida cotidiana y no se beneficiará suficientemente de ellos.

Lo que se pretende es centrar el foco de atención sobre la transferencia de los aprendizajes. Si queremos que un alumno se beneficie de un aprendizaje de un proceso de pensamiento tenemos que facilitarle, en primer lugar, las transferencias a otros aspectos de su vida, académicos o no, para que llegue a generalizarlo a cualquier situación que se le pueda presentar en su vida.

Para que el alumno realice estas trasferencias es fundamental que el profesor se lo facilite. La forma de facilitar que los aprendizajes sobre los procesos de pensamiento se transfieran es trabajar el control metacognitivo y el pensamiento reflexivo con las metodologías adecuadas.

En la experiencia que hemos llevado a cabo se utilizaron dos propuestas metodológicas bien fundamentadas de dos autores sobradamente conocidos, Feuerstein y Meichembaum, de lo que luego daremos cuenta.

Ahora bien, el problema sigue planteado: ¿enseñamos procesos de pensamiento dentro o fuera de las materias? Nuestra postura es intentar llegar a un punto medio: dentro y fuera. Proponemos enseñar procesos de pensamiento con materiales específicos fuera de las materias y con contenidos curriculares dentro de las asignaturas. Si bien el requisito que creemos es imprescindible para que esta experiencia tenga éxito es que sea el mismo profesor tutor el que realice ambas tareas.

De esta manera garantizamos, en la medida de lo posible, que los aprendizajes específicos realizados con materiales de "enseñar a pensar” se transferían a las áreas del currículum al utilizar el profesor la misma termino- 
logía, estrategias y metodología. Y de esta forma, una vez facilitada la primera transferencia de los procesos de pensamiento se favorecerá la transferencia a otras áreas y la generalización de los aprendizajes.

\section{3.- Metodologías facilitadoras de las transferencias.}

Como ya se ha indicado, las metodologías de "enseñar a pensar" no requieren unas formas de actuación complejas por parte del profesorado, sino mas bien un cambio de actitud hacia sus enseñanzas y los aprendizajes de los alumnos de tal forma que permitan a estos trabajar y elaborar sus propios pensamientos, fomentando el control metacognitivo y el pensamiento reflexivo en los alumnos, fijándonos no sólo en los resultados sino en el proceso que se ha seguido, $\mathrm{y}$ aprendiendo tanto, o más si cabe, de los errores como de los aciertos. En suma, el profesor debe dar a sus alumnos la oportunidad de pensar en cada situación de enseñanza y aprendizaje.

Dos son las metodologías de "enseñar a pensar” que nos han servido de referencia para fomentar estas actitudes en los profesores. Por un lado las estrategias de entrenamiento conductual cognitivo de Meichembaum (1977), utilizada fundamentalmente para el control de los procesos de pensamiento a través del lenguaje interior, fomentando la reflexibilidad y el autocontrol. Por otro la metodología de la mediación de Feuerstein (1986a, 1986b, 1988a, 1988b) como índice o guía de las actitudes docentes que son facilitadoras del control de los pensamientos por el propio sujeto, siendo el profesor el mediador de los aprendizajes como canalizador, seleccionador y presentador de la información. Pasamos a comentar brevemente ambas propuestas metodológicas.

\section{1.-Entrenamiento conductual cogni- tivo de Meichembaum}

Meichembaum (1977) señala la importancia del lenguaje interior como regulador del pensamiento y de la conducta en general. En este sentido se puede considerar a Meichembaum como el sintetizador de los presupuestos conductistas norteamericanos con las teorías cognitivistas provenientes de Rusia (Luria y Vigotski principalmente). Este autor presenta el lenguaje interior como controlador de la conducta. El lenguaje interior comprendería tanto el lenguaje verbal como las imágenes mentales constituyendo el vehículo que conforma las estructuras cognitivas.

El fallo en el procesamiento de la información por causa de falta de control en los metaprocesos (control sobre los propios procesos de pensamiento) hace que muchos sujetos tengan dificultades de aprendizaje . Este tipo de fallos se palia ejecutando la tarea de forma consciente con control verbal siguiendo los tres estadios que propone Luria en la iniciación y finalización de las conductas voluntarias:

-Primer estadio: el habla de los demás dirige y controla la conducta del educando.

-Segundo estadio: el habla manifiesta del educando es regulador y mediador de su propia conducta.

-Tercer estadio: el habla encubierta propia controla las acciones, adquiriendo el educando el rol de autogobierno.

Siguiendo estos planteamientos, las teorías del lenguaje interior de Meichembaum tienen su correlato metodológico que se concreta en los siguientes pasos:

1.- Modelado cognitivo: el profesor sirve de modelo, mientras ejecuta la acción con pensamento en voz alta.

2.- Guía externa manifiesta: el alumno realiza la acción con la guía verbal del profesor

3.- Autoguía manifiesta: el alumno realiza la acción con pensamiento en voz alta, dándose autoinstrucciones.

4.- Autoguía manifiesta atenuada: paulatinamente las instrucciones van siendo menos explícitas, pasando poco a poco al autocontrol mental. 
5.- Autoinstrucción encubierta: en el último paso el alumno se da autoinstrucciones no manifiestas con autocontrol mental del proceso.

Meichembaum ha desarrollado estrategias de entrenamiento en autoinstrucciones con la finalidad de que el niño sea capaz de regular su propia conducta para actuar de forma más eficaz. Se enseña a los niños el empleo de respuestas mediadoras que ejemplifican una estrategia general que permite controlar la conducta en diferentes circunstancias.

Asimismo Meichenbaum presenta una serie de sugerencias para favorecer la generalización de un programa de entrenamiento (tomado de Mayor, Suengas y González, 1993):

1.- El entrenamiento en estrategias cognitivas no ocurre rápidamente, sino que es un proceso prolongado que requiere una retroalimentación informativa. Los aprendices precisan tanto de la explicación de las estrategias como de la oportunidad de practicarla. Por lo tanto hay que adecuar los programas a la velocidad del aprendiz y asegurar la aplicación de las estrategias a diversas situaciones.

2.- Los aprendices deben desde el primer momento participar como sujetos activos en la generalización de las estrategias cognitivas, entendiendo el propósito y razones de tal aprendizaje. La retroalimentación informativa, además de indicarles el uso de la estrategia, debe indicarles el propósito que tiene y su utilidad.

3.- Las estrategias cognitivas han de ser lo más generales posibles para que puedan ser aplicadas a numerosas situaciones. Pero igualmente deben individualizarse para asegurar la compatibilidad con el aprendiz.

4.- Hay que enseñar al aprendiz a ser consciente de su proceso cognitivo, desautomatizando el proceso de aprendizaje y evitando usar las estrategias al modo de "receta”. Además de incorporar nuevas estrategias en su repertorio, debe aprender en qué tareas y entornos usarlas con sentimiento de competencia respecto a su eficacia personal.

5.- El entrenador debe ser alguien atractivo para el aprendiz, que merezca credibilidad y con el que pueda sentirse identificado. La persona que sirve de modelo debe compartir los pensamientos y sentimientos del aprendiz.

6.- Antes de empezar el entrenamiento hay que conocer la experiencia previa del aprendiz para basar los aprendizajes en las competencias ya adquiridas. Es necesaria la adquisición de ciertos prerrequisitos y regular el momento en el que se enseñan las estrategias metacognitivas.

7.- En el entrenamiento es importante graduar la dificultad. Se puede empezar en el ambiente habitual del aprendiz con tareas sencillas para paulatinamente ir encadenando aprendizajes más complejos. La graduación debe garantizar la trasferencia de una situación a otra, siendo evidentes los elementos comunes entre estrategias aprendidas y con los contextos de generalización.

8.- Se debe implicar activamente a los aprendices en las tareas de entrenamiento, evitando las repeticiones sin más de la tarea. Es necesaria una transformación mental de las estrategias que se están enseñando por parte del aprendiz y una ampliación de estas estrategias a su mundo personal y académico.

9.- Es necesario mantener el interés y la atención del aprendiz. Puede conseguirse manteniendo una relación positiva con el entrenador que presentando paulatinamente instrucciones, pistas, apoyos y utiliza un buen sistema de incentivos.

10.- El entrenamiento debe situarse en diversos entornos e implicar a varias personas para que se promueva el aprendizaje de las estrategias en situaciones variadas y contextos diferentes.

11.- Es necesario reforzar el uso de las estrategias alimentando el sentimiento de competencia y la satisfacción del aprendiz. El uso de estrategias metacognitivas en el 
entorno natural deben organizarse y favorecerse para reforzar estos sentimientos positivos.

\section{2.- Metodología de la mediación de Feuerstein}

Feuerstein (1986a, 1986b, 1988a, 1988b) en su la teoría modificabilidad estructural cognitiva, considera al organismo humano como un sistema susceptible de cambio y de modificación. La persona tiene capacidad propia para cambiar la estructura de su funcionamiento. Este es un enfoque de modificación activa que rechaza de plano la aceptación pasiva de la permanencia y estabilidad de los déficits cognitivos; por el contrario, este enfoque considera la capacidad intelectual de la persona como un proceso dinámico autorregulable que es capaz de dar respuesta a los estímulos ambientales.

El alumno puede modificar su patrón de desarrollo mediante una interacción activa entre él y las fuentes internas y externas de estimulación. Pero para que esta modificación se lleve realmente a cabo el sujeto ha de estar expuesto a una serie de experiencias de aprendizaje mediado en las que el mediador (padres y educadores fundamentalmente) tienen un papel fundamental en la selección, transmisión y organización de los estímulos. La modificabilidad es entendida como la modificación estructural del funcionamiento del individuo, que produce cambios en el desarrollo previsto. No se refiere a cambios específicos del comportamiento, sino a cambios estructurales que alteran el curso del desarrollo cognitivo. Esta modificación no ocurre al azar, sino por causa de una intervención intencional.

La modificabilidad del sujeto será producto de la interacción de experiencias y aprendizajes específicos orientados a producir cambios estructurales que alteran el curso del desarrollo, no en cuanto a sucesos aislados, sino a la manera de interaccionar y responder del organismo a las diferentes fuentes de información del medio ambiente. Estas expe- riencias que propician el desarrollo cognitivo es lo que denomina experiencias de aprendizaje mediado frente a la estimulación directa del medio.

La experiencia de aprendizaje mediado consiste en la transformación del estímulo producido por el medio a través de un agente, familiares y educadores generalmente. Este mediador selecciona, organiza, reordena, agrupa y estructura los estímulos en función de una meta específica. Estas experiencias a través de mediador proporcionan al sujeto una serie de estrategias y procesos que le llevarán a la formación de comportamientos que a su vez son prerrequisitos del buen comportamiento cognitivo.

El mediador ha de enseñar al sujeto el significado de la actividad más allá de las necesidades inmediatas, de forma que posteriormente este sujeto pueda anticipar la respuesta ante situaciones parecidas.

En todo este proceso de aprendizaje el sujeto forma parte activa al ser el procesador de la información y estar abierto a la modificabilidad cognitiva. (Feuerstein, 1973,1975).

Para el desarrollo cognitivo, según lo dicho, se ha de proporcionar al sujeto experiencias de aprendizaje mediado. Los estímulos los proporcionan los programas y actividades que se le presentan al alumno, pero para que estos estímulos sean efectivos habrá que realizar un proceso de mediación con una metodología específica que tiene las siguientes características según las indica Feuerstein:

\section{1.- Intencionalidad y reciprocidad}

Feuerstein define esta característica como la condición básica para llevar a cabo cualquier experiencia de aprendizaje. (Feuerstein, 1980).

Como dice Prieto, "el mediador trata de compartir las intenciones con el sujeto en un proceso mutuo que implica el conocimiento del enriquecimiento y desarrollo por parte de los dos” (Prieto, 1989; p. 36). 
La labor del mediador no es sólo la de presentar al sujeto los estímulos necesarios. Además de seleccionar y organizar la información el mediador ha de implicar al sujeto de forma que en él se produzcan cambios cuando procese y opere con dicha información. El alumno ha de percibir e interpretar los estímulos de forma significativa.

\section{2.- Trascendencia}

La mediación tiene que lograr que los aprendizajes que se produzcan lleguen más allá de la mera solución a los problemas que se plantean. Los aprendizajes que se produzcan no sólo han de servir para solucionar la situación presente, mientras se aplica el programa, sino que han de permitir que el sujeto sepa resolver otras situaciones futuras, y que, a su vez, le sirvan para realizar nuevos aprendizajes.

De esta forma el sujeto pensará en la tarea que está haciendo como algo importante para él, algo que va más allá de la mera solución al problema particular, algo sobre lo que merece la pena trabajar, descartando la información superflua y asimilando lo esencial.

La trascendencia de los conocimientos se concreta cuando se ponen en contacto las actividades y aprendizajes del pasado con el futuro, de tal forma que los conocimientos se generalicen a otras áreas y otros contenidos de la vida en general. podemos denominar a este proceso de establecimiento de trasferencias "generalización” o "puenteo" en términos de Feuerstein.

\section{3.- Significado}

Lo entendemos aquí en el mismo sentido en que Aussubel habla de aprendizaje con significado. El lo entiende como una forma eficaz de procesar la información que se da cuando se provoca en el sujeto una serie de conexiones entre los nuevos conocimientos y los ya existentes para que entiendan el proceso a través del cual se produce el conocimiento. (Ausubel, 1980).
Asimismo podríamos tomar las palabras de Bruner cuando dice que "el dominio cognitivo es recompensador especialmente cuando el aprendiz reconoce el poder acumulativo del conocimiento y cuando se percata de que aprender una cosa le permite pasar a algo que antes estaba fuera de su alcance y proseguir hasta obtener la perfección posible” (Bruner, 1978, p.78).

De esta forma el aprendizaje se debe a, por una parte la intencionalidad, y por otra la sustancialidad de la relación de tarea de aprendizaje con la estructura cognitiva.

Se trata de presentar la tarea haciendo despertar interés por ella; discutir la importancia de ésta y explicarle la finalidad que se pretende. Hay que implicar de forma activa y emocional al sujeto para que este se sienta motivado y no pierda el tiempo en realizar múltiples ensayos intentando dar con la respuesta correcta.

\section{4.- Competencia}

Entendemos este término no en el sentido de competitividad, sino en el de hacer que el sujeto se sienta competente para la realización de la tarea; o sea que se sienta capacitado para resolverlo. Se trata, por lo tanto, de fomentar en el alumno una positiva autopercepción y autoestima. Hay que potenciar el aprendizaje incluso cuando el sujeto se sienta incapaz de aprender.

Esto implica para el mediador la necesidad de organizar los aprendizajes de forma que se adapten a cada sujeto en particular, con la intención de que, según el nivel de cada individuo, se puedan resolver las tareas con éxito.

\section{5.- Regulación y control de la conducta}

Consiste en evitar la conducta impulsiva, o sea la emisión de la respuesta sin pensarla, desarrollando el pensamiento reflexivo o "insight". Se pretende que el sujeto realice su proceso de pensamiento a lo largo de tres fases: que obtenga la información de los conocimientos previamente adquiridos ("input”); que los utilice de forma coherente (elaboración); y que ex- 
prese se respuesta a través de un proceso de razonamiento (“output”).

El mediador ha de conseguir que el alumno se tome tiempo antes de emitir la respuesta para que pueda sopesarla y tenga en cuenta toda la información que posee. Se trata de que el sujeto llegue a conocer su propia forma de pensar y sus conocimientos y llegue a la trascendencia y al significado del aprendizaje.

6.- Participación activa y conducta compartida

Cuando se produce un aprendizaje mediado, el mediador comparte con los alumnos sus experiencias de forma que se sitúe en el lugar de éstos. De esta forma se facilitan las discusiones reflexivas ya que el aprendiz percibe al educador como uno más con el que se puede discutir un problema. Evidentemente el mediador ha de estar lo suficientemente preparado para saber encauzar la discusión en el sentido que interese, de forma que los alumnos puedan ir captando cual es el proceso de pensamiento adecuado para dar solución al problema.

En este sentido se evitará dar las soluciones de inmediato, fomentando en los sujetos un pensamiento autónomo y reflexivo que les lleve a razonar las respuestas. De esta forma el alumno actuará con independencia, sin tomar al pie de la letra y como verdad absoluta lo que el profesor diga.

Pero la conducta compartida no solo se refiere a la relación profesor-alumno, sino también a la colaboración que puede establecerse entre los alumnos. En este sentido el mediador ha de fomentar el trabajo cooperativo enseñando a los alumnos que existen puntos de vista diferentes al suyo que hay que respetar y sobre los que se puede discutir con buenos razonamientos intentando dar solución a los problemas. Es lo que Feuerstein denomina "pensamiento divergente".

7.- Individualización y diferenciación psicológica
Sabemos que cada individuo es único y diferente, es un principio elemental en educación. Aquí se trata de aceptar este hecho teniendo en cuenta que cada uno puede pensar de forma distinta. Se trata de aplicar modelos de aprendizaje según las diferencias individuales y dependiendo de los diferentes estilos cognitivos.

A su vez, hay que fomentar en el sujeto respuestas divergentes, potenciando el pensamiento independiente de forma que se desarrolle un aprecio por los valores individuales y una diferenciación psicológica sin olvidar a los otros.

8.- Mediación de la búsqueda, planificación y logro de los objetivos de la conducta.

El mediador ha de conseguir que los sujetos orienten su atención hacia la consecución de metas a corto y largo plazo, superando las necesidades inmediatas del momento.

En este aspecto se van a ver implicados procesos superiores de pensamiento, así como mecanismos de autorregulación y autocorrección de la conducta.

El mediador ha de conseguir que los alumnos establezcan metas individuales que exijan ser perseverantes y diligentes para lograrlas. Se les pedirá que establezcan un plan, y por lo tanto, habrán de saber discriminar entre las metas reales y las irreales, a la vez que han de aprender a ser flexibles y a saber modificar los objetivos en función de las necesidades del momento.

9.- Mediación del cambio: búsqueda de la novedad y complejidad

El mediador ha de fomentar en los alumnos la curiosidad intelectual, el pensamiento divergente. Tienen que aprender a buscar lo novedoso de las tareas. Igualmente aprenderán a ser autocríticos sometiendo sus ideas a la opinión de los compañeros, viendo como se puede solucionar un problema de formas distintas y como se puede aumentar la complejidad de éste. También es labor del mediador el presen- 
tar las tareas de forma novedosa utilizando diferentes recursos, metodologías, distintas modalidades de lenguaje, etc.

10.- Mediación del conocimiento de la modificabilidad y del cambio

El alumno ha de llegar a autopercibirse como sujeto activo a la hora de poder modificar su funcionamiento cognitivo. Ha de ser consciente de que es capaz de generar y procesar información y de que así mismo es capaz de cambiar su forma de pensar.

Para que el sujeto llegue a tener un conocimiento objetivo de sí mismo y de su potencial de cambio cognitivo se ha de fomentar en él el pensamiento reflexivo y la interiorización de los sucesos. Se han de desarrollar en el alumno los mecanismos de autorregulación y control de la conducta.

\section{4.-Un ejemplo: estudio empírico del aprendizaje de estrategias metacogni- tivas y transferencias.}

Vamos a ilustrar estos comentarios anteriormente hechos con una experiencia en la práctica educativa del aula. Para ello hemos realizado un modesto estudio empírico con el que pretendemos aportar algunos datos que corroboren nuestras afirmaciones, mas que intentar obtener grandes conclusiones ampliamente generalizables

El objetivo general del estudio era demostrar la importancia de una correcta metodología en la enseñanza de los procesos de pensamiento. Como ya hemos puesto de manifiesto, pensamos que un profesor entrenado en las metodologías de "enseñar a pensar" facilita el aprendizaje de estrategias metacognitivas y las transferencias a los alumnos cuando utiliza instrumentos específicos para la enseñanza de estos procesos, frente a un profesor que utilice metodologías tradicionales con materiales habituales en las aulas.

Por lo tanto dos eran los objetivos específicos de este estudio: a) Evaluar la influencia de la metodologías de "enseñar a pensar" (la mediación y conductual cognitiva) en el aprendizaje de estrategias metacognitivas.

b) Evaluar la influencia de la metodología de "enseñar a pensar" en las transferencias realizadas por los alumnos.

Para este estudio utilizamos un diseño con un grupo experimental y un grupo de control, de dos centros públicos de Salamanca capital, pertenecientes ambos a la misma zona y sin diferencias culturales o socio-económicas entre ellos. Con un nivel cultural medio, los padres tienen estudios primarios y medios, trabajan por cuenta ajena o son funcionarios mayoritariamente. Es un barrio periférico de clase media no especialmente conflictivo y bien desarrollado en infraestructuras.

La muestra quedó compuesta por 47 alumnos de los que 25 pertenecían al grupo experimental y 22 al grupo de control. La edad de los sujetos oscilaba alrededor de los 10 años. Ambos grupos fueron seleccionados por no presentar problemas destacables ni en cuanto a dificultades en los aprendizajes ni en conducta, aun siendo suficientemente heterogéneos.

En el grupo experimental se aplicaron tres sesiones semanales con los materiales de “enseñar a pensar” siguiendo la metodología de la mediación y el entrenamiento conductual cognitivo. En el grupo de control el tutor empleaba tres sesiones equivalentes que denominamos "resolución de problemas", con una metodología tradicional no específica centrado fundamentalmente en el área de matemáticas. Estos programas fueron aplicados durante un curso académico.

En el grupo experimental se utilizaron materiales de varios programas editados y sobradamente conocidos, se hizo una selección en función de la adecuación de los materiales a la edad y nivel académico de los alumnos y que tuvieran relación con procesos utilizados en la resolución de problemas. Los programas que se han utilizado son: 
- Programa de Enriquecimiento Instrumental (R. Feuerstein, 1992)

- Proyecto Inteligencia "Harvard” (Megía

Fernández, 1993)

- Programa de Intervención Educativa para Aumentar la Atención y la Reflexibidad (Gargallo, 1993)

El objetivo de estos programas es mejorar procesos básicos de pensamiento: atención, comparaciones, clasificaciones, orientación espacial, pensamiento hipotético, pensamiento analítico, razonamiento lógico, etc. Se pretende asimismo mejorar el proceso de pensamiento enseñando al sujeto a estructurar y planificar sus procesos con un paulatino incremento del autocontrol. Se utilizan las metodologías anteriormente descritas, dando especial importancia al pensamiento reflexivo y a las transferencias y generalizaciones de los procesos aprendidos.

\section{1.-Evaluación de la metacognición y de las transferencias}

Evaluar los procesos cognitivos subyacentes a la realización de una tarea es una área difícil. Como señalan Mayor, Suengas y González (1993), es uno de los grandes problemas con los que se enfrenta la investigación. Operacionalizar la actividad metacognitiva es una tarea difícil, ya que ésta no se presenta como una respuesta observable, sino que debemos inferirla a partir de procedimientos y sistemas indirectos.

Los procedimientos más frecuentemente empleados, señalados por estos autores, son los informes verbales (autoinformes del sujeto mientras realiza la tarea), la observación (bien sea del pensamiento en voz alta, de escenificaciones o de la ejecución de la tarea) y algunas escalas e instrumentos de evaluación. Siguiendo la propuesta de estos autores elaboramos un cuestionario que nos permitiese evaluar la actividad metacognitiva de los sujetos (Mayor, Suengas y González, 1993, p.171-178) que contempla 45 variables agrupadas en los siguientes aspectos:

\section{1.- Componentes cognitivos}

1.2.- Tareas (problemas/ objetivos)

1.3- Modos (rasgos/ características)

2.- Control

2.1- Componentes de control

2.2.- Tareas o problemas

2.3.- Modos de control

3.- Autopoiesis

3.1.- Componentes de autopoiesis

3.2.- Tareas

3.3.- Modos

4.- Variables de la metacognición del sujeto

5.- Variables de la metacognición del contexto

6.-Variables de la metacognición de la actividad.

Para evaluar las transferencias a la resolución de problemas elaboramos un cuestionario con tres ejercicios de problemas, no específicamente matemáticos, con una secuencia paso a paso que permitieran al alumno poner de manifiesto si utilizaba las estrategias planteadas en los programas aplicados. Para elaborar este instrumento tomamos como referencia las aportaciones de Martinez Beltrán (1990) y las de Prieto $(1989,1993)$ en sus instrumentos elaborados para evaluar la eficacia de la aplicación del Programa de Enriquecimiento Instrumental.

Los pasos que tenían que seguir para resolver los problemas nos otorgaban las variables que evaluamos, concretadas en:

- Lectura y comprensión de instrucciones.

- Exploración sistemática de los datos.

- Consideración de datos relevantes.

- Consideración de varias fuentes de información.

- Utilización de principios de clasificación y /o categorización.

- Estrategias de pensamiento lógico.

- Estrategias de pensamiento hipotético.

- Consideración de varias alternativas.

- Comprobación.

\section{1.- Toma de conciencia}


- Comunicación de los resultados clara y explícita.

Con ambas pruebas se evaluaron los dos grupos antes y después de la aplicación de los programas, lo que nos permitió estudiar las diferencias entre el grupo experimental y el de control como explicamos a continuación.

\section{2.- Resultados y análisis.}

Para comprobar la equivalencia de ambos grupos al inicio del trabajo se realizó un ANOVA entre ambos grupos en el que se obtuvieron valores de probabilidad altos en ambas escalas (metacognición $\mathrm{p}=$ 0,852 transferencias $\mathrm{p}=0,591$ ) lo que implicaba que los grupos, experimental y control eran semejantes y no diferían significativamente al inicio del estudio.

Una vez aplicados los tratamientos se realizó nuevamente un ANOVA con los resultados de la evaluación final de ambos grupos, obteniendo valores de probabilidad significativos (metacognición $\mathrm{p}=0,001$ transferencias $\mathrm{p}=0,001$ ) lo que confirmaba nuestra hipótesis en el sentido de que una intervención por parte del profesor tutor con materiales de "enseñar a pensar" siguiendo una metodología específica beneficia el aprendizaje de estrategias metacognitivas y la transferencia de estas a otras áreas, en nuestro caso en resolución de problemas.

Ahora bien, aún habiendo diferencias significativas entre ambos grupos, esto no quiere decir que el grupo de control no haya mejorado ya que sus puntuaciones medias se elevaban tras la intervención. Realizamos varias pruebas de contraste de medias para comprobar si era significativa la ganancia de cada uno de los grupos en cada una de las escalas.

De esta forma encontramos en el grupo de control que en la escala de metacognición, aún habiendo ganancia, esta no es significativa $(\mathrm{T}=.589, \mathrm{p}=0,559)$ sin embargo sí es significativa en el grupo experimental ( $\mathrm{T}=$ $4,362, p=0,000$ ).

En la escala de transferencias ocurre exactamente igual, $\mathrm{T}=.815, \mathrm{p}=0,419$ para el grupo de control y $\mathrm{T}=3979, \mathrm{p}=0,000$ para el experimental.

\section{5.- Conclusiones.}

El estudio realizado, como hemos titulado, se trata de un sencillo ejemplo con una muestra pequeña del que no deben extraerse grandes conclusiones, como hemos indicado.

Sin embargo, a pesar de esto sí presenta algunos datos cuantitativos interesantes que avalan nuestra hipótesis y nos corroboran nuestro enfoque. Efectivamente, las metodologías específicas de los programas de "enseñar a pensar” facilitan la enseñanza de estrategias metacognitivas y la transferencia de éstas a otros ámbitos académicos o extraacadémicos.

Así mismo, hemos considerado desde el principio del estudio que la situación ideal era la aplicación de los programas por los mismos profesores que imparten las distintas materias. Si bien el que todo el profesorado conozca los programas es inviable, sí puede ser importante que adquieran una metodología, o mejor una "filosofía de trabajo" que suponga un cambio de actitud hacia la enseñanza de los procesos de pensamiento, estén o no ligados a programas específicos o a las materias académicas.

Lo que no reflejan las cifran anteriormente expuestas y que, de alguna manera, ha sido percibido con cierta claridad por los observadores, es la diferente actitud de ambos profesionales, a los que estamos sinceramente agradecidos, que se sometieron a la dureza de una crítica más allá de una evaluación formal del profesorado.

En este sentido se pudo observar que la diferencia radicaba en la distinta actitud ante la situación de enseñar a resolver problemas. El 
profesor no preparado específicamente repetía los esquemas que él, a su vez, había aprendido como alumno. Esto es lo que hacemos todos los profesionales de la educación cuando no se nos enseñan metodologías alternativas de una forma activa. Esta actitud hacía perder grandes oportunidades en sus alumnos de que pensaran. Un ejemplo aclarará este comentario. Así, cuando los alumnos habían terminado un ejercicio, el profesor lo corregía, en un primer momento, indicando sólo aquellos que estaban bien o mal, dando tácitamente una valoración sólo del resultado, y pedía al alumno que intentara de nuevo repetir los ejercicios fallidos. Esta situación se repetía en varias ocasiones hasta que el profesor explicaba al alumno cómo solucionar los ejercicios exponiéndole su forma de resolverlo (la del profesor, no la del alumno).

Esta situación es una perdida de oportunidad de que el alumno aprenda a pensar. El otro profesional, en situaciones semejantes utilizaba con los alumnos lo que podriamos llamar un "método socrático", centrándose más en el fallo que en el acierto y haciendo reflexionar al alumno sobre su proceso de pensamiento; es decir, trabajando la metacognición con pensamiento reflexivo en busca del "eureka" que hace que el alumno se de cuenta de dónde comete el error.

En cualquier caso, para que estas afirmaciones fueran más tajantes debemos plantearnos seguir investigando con muestras más representativas, de diferentes ámbitos, y en diferentes situaciones.

Así mismo debemos insistir en que, a demás de la investigación educativa y los debates científicos que permiten ir avanzando en el conocimiento de la educación, se debe trabajar con los profesionales, formación del profesorado, para que conozcan metodologías, estrategias, aprendan recursos novedosos y adopten actitudes favorables hacia el aprendizaje de los alumnos.

\section{BIBLIOGRAFIA}

Aussubel, D. (1980) Psicología de la Educación. Un punto de vista cognoscitivo. Trillas. México

Banyard, P.; Cassels, A. y otros (1995). Introducción a los procesos cognitivos. Ariel. Barcelona.

Beltrán, J. (1993) Procesos, estrategias y técnicas de aprendizaje. Madrid, Síntesis

Bruner, G. (1978). El proceso mental de aprendizaje. Narcea. Madrid

Ewey,J. (1989) Cómo pensamos. Paidos. Barcelona.

Feuerstein, R. (1979) The dinamic assesment of retarded performer: The Learning Potential Assesmente Decive, teory, instrumentes and techniques. University Park Press. Baltimore.

Feuerstein, R. (1986a) Mediated learning Experience. Hadassah-Wizo-Canada Research Institute. Jerusalem.

Feuerstein, R. (1986b) "Experiencia de Aprendizaje Mediado” Siglo Cero, 106, 2832.

Feuerstein, R. (1988a). "La Teoría de la Modificabilidad Estructural Cognitiva”. Revista de innovación e Investigación Educativa, 4, 7-14.

Feuerstein, R. (1988b). "La Experiencia de Aprendizaje Mediado y el Funcionamiento Cognitivo". Revista de innovación e Investigación Educativa, 4, 15-20.

Feuerstein, R. (1988c). "El Programa de Enriquecimiento Instrumental y la Evaluación del Potencial de Aprendizaje". Revista de innovación e Investigación Educativa, 4, 21-26.

Feuerstein, R. (1992) Programa de Enriquecimiento Instrumental. Bruño. Madrid.

Feuerstein, R. and Rand, Y. (1977). Studies in cognitive modificability Instrumental Enrichment Redevelopment of cognitive functions of retarded early adolescents. Hadassah-Wizo-Canada Research Institute, Jerusalem.

Feuerstein, R., Rand, Y. Hoffman, M.B. and Miller, R. (1980). Instrumental Enrichment. University Park Press. Baltimore.

Feuerstein, R., Rand, Y. Hoffman, M.B. and Miller, R. (1980). The Dinamic Asessment 
of Retarded Performers. University Park Press. Baltimore

Feuerstein, R.; Klein, S.; Tannenbaum, A.J. (1991) Mediated Learning Experience: Theoretical, psichosocial and learning implications. Freund Publishing House. London.

Gargallo, B. (1993) Programa de Intervención Educativa para Aumentar la Atención y la Reflexiblilidad. TEA, Madrid

González Mas, R. (1978) Actividades adiestradoras de las funciones mentales superiores. Científico -Técnica. Madrid

M.E.C. (1989a). Libro Blanco para la Reforma del Sistema Educativo. Madrid, Ministerio de Educación y Ciencia.

M.E.C. (1989b). Diseño Curricular Base. Madrid, Ministerio de Educación y Ciencia.

Martín Izard, J.F. (1995). “Aplicación del Programa de Enriquecimiento Instrumental a un grupo de sujetos pre-operacionales”. Revista de Educación Especial, $n^{\circ} 19,21-$ 37.

Martín Izard, J.F. (1997). “La Mediación en los Programas de "Enseñar a Pensar" como actos didácticos interactivos “. Revista Aula vol.9 123-137.

Martín Izard, J.F. (1999). “Tipologías de sujetos según los perfiles de disfunciones cognitivas “. Revista Española de Orientación y Psicopedagogía, vol. $10 n^{\circ}$ 17. 99113.

Martín Izard, J.F.(2000) "Evolución de las disfunciones cognitivas al aplicar el P.E.I." Revista Bordón vol 52., nª3 407-419

Martín Izard, J.F.(2001) "La adaptación social, el pensamiento autorreferencial y la mejora de la inteligencia.”. Revista de Educación Especial n²0, 77-91

Martínez Beltrán, J. M. y otros (1990) Metodología de la mediación en el PE.I.. Bruño, Madrid.

Mayor, Suengas y González, 1993. Estrategias Metacognitivas. Aprender a aprender y aprender a pensar. Madrid, Síntesis

Megía Fernández, M. (1993). Proyecto de Inteligencia "Harvard". Cepe, Madrid
Meichembaum, D. (1977). CognitiveBehavior modification: An integrative approach. Plenum Press. New York.

Meichembaum, D. y Goodman J. (1971). "Training impulsive chuildren to talk to themselves. A means of developimg selfcontrol”. Journal of Abnormal Psychology, 77, 115-126

Monereo y otros (1996) "Orientación y tutoría educativas en el ámbito de las estrategias de aprendizaje”, en M. Alvarez y R. Bisquerra (coords.) Manual de orientación y tutoría. Barcelona, Praxis

Monereo, C. y Castelló (1990) Las estrategias de aprendizaje. Barcelona, Edibé.

Nickerson, R.S.; Perkins, D.N.; Smith, E.E. (1987) Enseñar a pensar. Aspectos de la amplitud intelectual, Paidos ,Barcelona.

Presley, M. and others (1990) A primer of research on cognitive strategy instruction. The important issues and how to address them. Educational Psychology Review, (2) ,1, 1-58

Prieto Sánchez, M.D. (1987) El potencial de aprendizaje: Un modelo y un sistema aplicado a la evaluación. I.C.E. Universdad de Murcia

Prieto Sánchez, M.D. (1988) "Evaluación de las capacidades cognitivas y metacognitivas del potencial de aprendizaje”. Revista de innovación e Investigación Educativa, 4, 27-58.

Prieto Sánchez, M.D. (1989) Modificabilidad Estructural Cognitiva y P.E.I. Bruño. Madrid.

Prieto Sánchez, M.D. y Pérez Sánchez, L. (1993) Programas para la mejora de la inteligencia. Teoría , aplicación y evaluación.. Síntesis. Madrid

Wittrock, M. C. (1985). A constructive review of research on learning strategies. En C. Weinstein and others. Learning and Study Stratagies. San Diego, Academic

Yuste Herranz, C. (1997). Programas de mejora de la inteligencia. CEPE. Madrid 
Martín Izard, J. F. (2001). Enseñanza de procesos de pensamiento: Metodología, metacognición y transferencias. RELIEVE, v. 7, n. 2, p. 73-88. http://www.uv.es/RELIEVE/v7n2/RELIEVEv7n2_2.htm

\section{ARTICLE RECORD / FICHA DEL ARTÍCULO}

\begin{tabular}{|c|c|}
\hline $\begin{array}{l}\text { Reference / } \\
\text { Referencia }\end{array}$ & $\begin{array}{l}\text { Martín Izard, J. F. (2001). Enseñanza de procesos de pensamiento: Metodología, metacognición y trans- } \\
\text { ferencias. RELIEVE, vol. 7, n. 2. Consultado en www.uv.es/RELIEVE/v7n2/RELIEVEv7n2_2.htm en } \\
\text { (poner fecha). }\end{array}$ \\
\hline $\begin{array}{l}\text { Title / } \\
\text { Título }\end{array}$ & Enseñanza de procesos de pensamiento: Metodología, metacognición y transferencias. \\
\hline $\begin{array}{l}\text { Authors / } \\
\text { Autores }\end{array}$ & Juan Francisco Martín Izard \\
\hline $\begin{array}{l}\text { Review / } \\
\text { Revista }\end{array}$ & Revista ELectrónica de Investigación y EValuación Educativa (RELIEVE), v. 7, n. 2 \\
\hline ISSN & $1134-4032$ \\
\hline $\begin{array}{l}\text { Abstract / } \\
\text { Resumen }\end{array}$ & $\begin{array}{l}\text { We analyze the importance of teaching with the correct methodology in thinking processes. We also } \\
\text { present an empirical study which shows the importance of teachers' familiarity with these methodolo- } \\
\text { gies of thinking processes. } \\
\text { Analizamos la importancia de una correcta metodología en la enseñanza de los procesos de pensamiento. } \\
\text { Presentamos también un estudio empírico en el que se intentará poner de manifiesto la importancia de } \\
\text { que el profesor conozca las metodologías mencionadas }\end{array}$ \\
\hline $\begin{array}{l}\text { Keywords / } \\
\text { Descriptores }\end{array}$ & $\begin{array}{l}\text { Thinking processes, metacognition, transference. } \\
\text { Procesos de pensamiento, metacognición, transferencia }\end{array}$ \\
\hline $\begin{array}{l}\text { Institution / } \\
\text { Institución }\end{array}$ & Universidad de Salamanca (España) \\
\hline $\begin{array}{l}\text { Publication } \\
\text { site / } \\
\text { Dirección }\end{array}$ & http://www.uv.es/RELIEVE \\
\hline $\begin{array}{l}\text { Language / } \\
\text { Idioma }\end{array}$ & Spanish (Title, abstract and keywords in English ) \\
\hline
\end{tabular}

\section{Revista ELectrónica de Investigación y EValuación Educativa (RELIEVE)}

[ ISSN: 1134-4032 ]

(C) Copyright, RELIEVE. Reproduction and distribution of this articles it is authorized if the content is no modified and their origin is indicated (RELIEVE Journal, volume, number and electronic address of the document).

(C) Copyright, RELIEVE. Se autoriza la reproducción y distribución de este artículo siempre que no se modifique el contenido y se indique su origen (RELIEVE, volumen, número y dirección electrónica del documento). 